\title{
POINCARÉ-LEFSCHETZ DUALITY FOR THE HOMOLOGY CONLEY INDEX
}

\author{
CHRISTOPHER MCCORD
}

\begin{abstract}
The Conley index for continuous dynamical systems is defined for (one-sided) semiflows. For (two-sided) flows, there are two indices defined: one for the forward flow; and one for the reverse flow. In general, the two indices give different information about the flow; but for flows on orientable manifolds, there is a duality isomorphism between the homology Conley indices of the forward and reverse flows. This duality preserves the algebraic structure of many of the constructions of the Conley index theory: sums and products; continuation; attractor-repeller sequences and connection matrices.
\end{abstract}

\section{INTRODUCTION}

The Conley index for continuous dynamical systems is defined for isolated invariant sets in local semiflows. A local semiflow on a Hausdorff space $X$ consists of a neighborhood $U$ of $X \times\{0\}$ in $X \times \mathbf{R}^{+}$and a map $\mu: U \rightarrow X$ such that $\mu_{0}=$ id and $\mu_{s} \circ \mu_{t}=\mu_{s+t}$. If $S$ is isolated in $X$, it has index pairs $(N, L)$ defined, with the homotopy type $[N / L]$ well-defined independent of the index pair chosen. This defines the Conley index $I(X ; S)$ of $S$ in $X$, and after passing to homology, the homology Conley index $C H_{*}(X ; S)$. The Conley index theory developed from these objects has proved to be a valuable tool for the study of dynamical systems in a number of settings (cf. $[1-4,8,9])$.

If the space has a (two-sided) flow $\phi: V \rightarrow X$, where $V$ is a neighborhood of $X \times\{0\}$ in $X \times \mathbf{R}$, there are two local semiflows defined: $U_{1}=V \cap\left(X \times \mathbf{R}^{+}\right)$, $\mu_{1}=\phi \mid U_{1}$; and $U_{2}=($ id, - id $)\left(V \cap\left(X \times \mathbf{R}^{-}\right)\right), \mu_{2}=\phi \circ($ id,,- id $)$. These will be referred to as the forward and reverse flows, respectively. Any isolated invariant set in $X$ then has two Conley indices defined: its index in the forward flow, and its index in the reverse flow. These are not equal, yet in many cases, they provide the same information about the flow. In this paper, the differences and similarities between the homology and cohomology indices in the forward and reverse flows are examined.

The main result, Theorem 2.1, shows that for flows on an orientable manifold, the indices for the forward and reverse flows are related by a Poincaré-Lefschetz duality isomorphism. This duality is well-defined (i.e. independent of the index

Received by the editors November 4, 1989.

1980 Mathematics Subject Classification (1985 Revision). Primary 55U30, 34C35; Secondary $55 \mathrm{~N} 20,58 \mathrm{~F} 25$.

Key words and phrases. Conley index, homology Conley index, Poincaré duality.

Work supported in part by DARPA Applied and Computational Mathematics Program. 
pairs chosen) and preserves the algebraic structure of many of the constructions of the Conley index theory: sums; products; continuation; attractor-repeller sequences; connection matrices and Morse decompositions. In particular, duality shows that any dynamical information obtained from these algebraic constructions can be obtained equally from the forward and reverse flows. This can be summarized as follows:

Given a property of flows on orientable manifolds which can be detected by the group structure of the (co)homology Conley indices of isolated invariant sets of a single flow or parameterized family of flows, the homology and cohomology indices for the forward and reverse flows all provide equivalent information about the property. When other classes of flows or other algebraic structures are considered, the four indices may provide distinct information.

Section 1 describes the main elements of the Conley index theory, and presents some examples which will be used to illustrate the results. In $\S 2$, the duality isomorphism is constructed and shown to be well defined. In $\S 3$, the isomorphism is shown to commute with sums, products, continuation, and attractorrepeller sequences, and to conjugate connection matrices and Morse inequalities. Some simple examples are considered to illustrate the properties of the index not preserved by duality. Finally, some of the dynamical applications of duality are considered in $\S 4$.

\section{The Conley indeX}

We begin with a brief survey of the index theory, using the notation of [10]. The reader is referred to $[1,6,7,13]$ for more complete developments of the theory.

We will assume that $M$ is a locally compact manifold with boundary with a complete $C^{1}$ flow defined on it. A compact set $S \subseteq M$ is an isolated invariant set if there exists a compact neighborhood $N$ of $S$ such that $S$ is the maximal invariant set of $N S=\{x \in N: x \cdot \mathbf{R} \subseteq N\}$. Such an $N$ is an isolating neighborhood for $S$. The Conley index studies isolated invariant sets; the essential tool for this study being an index pair for $S$ in $M$ - a compact pair $(N, L)$ satisfying the following axioms:

(i) $\overline{N \backslash L}$ is an isolating neighborhood for $S$.

(ii) $L$ is positively invariant in $N$ : if $x \in L, x \cdot[0, t] \subseteq N$, then $x \cdot t \in L$.

(iii) $L$ is an exit set for $N$ : if $x \in N, x \cdot \mathbf{R}^{+} \nsubseteq N$, then there exists a $t>0$ such that $x \cdot[0, t] \subseteq N, x \cdot t \in L$.

In $[1,4,12,13]$ the basic properties of index pairs are established. These are that index pairs exist for all isolated invariant sets in locally compact metric flows, and that all index pairs for $S$ in $M$ have homotopic quotient spaces $N / L$. As we will be interested in comparing the indices of $S$ in the forward and reverse flows, it will be convenient to work with a special class of index pairs, similar to those developed in [12].

Given $S$, let $N$ be an isolating neighborhood of $S$ in $M, N_{0}=N \cap \partial M$, $N_{1}$ the "immediate entrance set" $\{x \in \partial N: \forall \varepsilon>0, x \cdot[\varepsilon, 0] \nsubseteq N\}, N_{2}$ the "immediate exit set" $\{x \in \partial N: \forall \varepsilon>0, x \cdot[0, \varepsilon] \nsubseteq N\}$. The quadruple $\left(N, N_{0}, N_{1}, N_{2}\right)$ can be chosen so that $\partial N=N_{0} \cup N_{1} \cup N_{2}$, and so that $N$, $N_{0}, N_{1}, N_{2}$ are all topological manifolds with boundary. Let $\mathscr{M}(M, \partial M ; S)$ denote the set of such quadruples for $S$ in $M$. Then $\left(N, N_{1}\right)$ is an index pair 
for $S$ in $M$ in the reverse flow; $\left(N, N_{2}\right)$ is an index pair for $S$ in the forward flow.

An explicit formula for the homotopy equivalence of index pairs in terms of the flow is given in [13]. Given $\left(N, N_{0}, N_{1}, N_{2}\right),\left(N^{\prime}, N_{0}^{\prime}, N_{1}^{\prime}, N_{2}^{\prime}\right) \in$ $\mathscr{M}(M, \partial M ; S)$, the index pairs of the reverse flow are related by the homotopy equivalence $\phi_{-}:\left(N / N_{1}, N_{0} / N_{1}\right) \rightarrow\left(N^{\prime} / N_{1}^{\prime}, N_{0}^{\prime} / N_{1}^{\prime}\right)$, given by

$$
\phi_{-}[x]= \begin{cases}{[x \cdot-3 T],} & x \cdot[-2 T, 0] \subseteq N \backslash N_{1}, x \cdot[-3 T,-T] \subseteq N^{\prime} \backslash N_{1}^{\prime}, \\ {\left[N_{1}^{\prime}\right],} & \text { otherwise, }\end{cases}
$$

for $T$ sufficiently large. Likewise for the index pairs of the forward flow, there is a homotopy equivalence $\phi^{+}:\left(N / N_{2}, N_{0} / N_{2}\right) \rightarrow\left(N^{\prime} / N_{2}^{\prime}, N_{0}^{\prime} / N_{2}^{\prime}\right)$ given by

$$
\phi^{+}[x]= \begin{cases}{[x \cdot 3 T],} & x \cdot[0,2 T] \subseteq N \backslash N_{2}, x \cdot[T, 3 T] \subseteq N^{\prime} \backslash N_{2}^{\prime}, \\ {\left[N_{2}^{\prime}\right],} & \text { otherwise }\end{cases}
$$

The quotient spaces $N / N_{1}$ for the reverse flow and $N / N_{2}$ for the forward flow, together with these maps, form categories $I^{-}(M ; S)$ and $I^{+}(M ; S)$ respectively, which define the Conley index of $S$ in $M$ for the reverse and forward flows (see [13] for details). The categories are also inverse systems, ordered by the inclusion $N^{\prime} \subseteq N$. Applying one of the functors $H^{*}, H_{*}$ to one of the categories $I^{+}, I^{-}$generates an inverse system of (isomorphic) groups, with the (co)homology Conley index groups $C H_{+}^{*}(M ; S), C H_{-}^{*}(M ; S), C H_{*}^{+}(M ; S)$, $\mathrm{CH}_{*}^{-}(\mathrm{M} ; \mathrm{S})$ the inverse limits of the systems.

As every neighborhood of $S$ contains the total space $N$ of an index quadruple, the subcategories of index pairs derived from index quadruples are cofinal with the total categories. Thus, for the purposes of this paper, the homology Conley index can be viewed as an inverse limit of (isomorphic) homology groups derived from index quadruples. That is, for any homology theory $h_{*}$, the cohomology Conley index $\mathscr{C} h_{*}^{+}(M ; S)$ for the forward flow are represented by $h_{*}\left(N, N_{2}\right)$; the index $\mathscr{C} h_{*}^{-}(M ; S)$ for the reverse flow is represented by $h_{*}\left(N, N_{1}\right)$.

Example 1.1. Suppose $M$ is an orientable $n$-manifold with a flow, $S$ a fixed point or periodic orbit, or more generally a compact orientable invariant submanifold of $M$. Further, suppose $S$ is normally hyperbolic in the flow (i.e. there is a splitting of the tangent bundle $T_{S} M \cong T S \oplus E^{s} \oplus E^{u}$ and constants $C, a>0$ such that for all $t>0,\left\|D \mu_{t} \mathbf{v}\right\|<C e^{-a t}\|\mathbf{v}\|$ for $\mathbf{v} \in E^{s}$ and $\left\|D \mu_{t} \mathbf{v}\right\|>C^{-1} e^{a t}\|\mathbf{v}\|$ for $\left.\mathbf{v} \in E^{u}\right)$. If $E^{s}$ has dimension $s$ and $E^{u}$ has dimension $u$, there is a tubular neighborhood $E$ of $S$ in $M$ and fibration $\mathbf{R}^{s} \times \mathbf{R}^{u} \rightarrow E \rightarrow S$ with the following properties:

(i) $E$ and $S$ have flows inherited from $M$;

(ii) The projection map $E \rightarrow S$ is equivariant with respect to these flows;

(iii) The flow on $E$ is linear on fibers, contracting on $\mathbf{R}^{s}$, expanding on $\mathbf{R}^{u}$. An index pair for $S$ in the forward flow is then a pair $\left(N, N_{2}\right)$ with fibration

$$
\left(D^{s} \times D^{u}, D^{s} \times S^{u-1}\right) \rightarrow\left(N, N_{2}\right) \rightarrow S .
$$

Likewise, an index pair for $S$ in the reverse flow is a pair $\left(N, N_{1}\right)$ with fibration

$$
\left(D^{s} \times D^{u}, S^{s-1} \times D^{u}\right) \rightarrow\left(N, N_{1}\right) \rightarrow S .
$$


If $S$ is a single critical point, the forward and reverse indices have the homotopy types of spheres: $I^{+}(M ; S)=\Sigma^{u}:=\left[S^{u}\right] ; I^{-}(M ; S)=\Sigma^{s}$. That is,

and

$$
C H_{-}^{k}(M ; S)= \begin{cases}\mathbf{Z}, & k=u, \\ 0, & k \neq u,\end{cases}
$$

$$
C H_{k}^{+}(M: S)= \begin{cases}\mathbf{Z}, & k=s=n-u, \\ 0, & k \neq s .\end{cases}
$$

It is trivial then that $C H_{-}^{k}(M ; S) \cong C H_{n-k}^{+}(M ; S)$.

More generally, if $S$ is a compact orientable manifold and one (hence both) of the fibrations above is orientable, the forward and reverse homology indices are just suspensions (of degree $u$ and $s$ respectively) of the homology of $S: C H_{-}^{k}(M ; S) \cong H^{k-u}(S)$ and $C H_{n-k}^{+}(M ; S) \cong H_{k-s}(S)$. As $S$ is a compact orientable manifold of dimension $n-u-s$, we can apply Poincaré duality to the homology of $S$ and obtain an isomorphism $H^{k-u}(S) \cong H_{n-k-s}(S)$. Composing these yields $C H_{-}^{k}(M ; S) \cong C H_{n-k}^{+}(M ; S)$. The goal of this paper will be to establish this duality in much greater generality. The following examples will be used in $\S 3$ to illustrate some of the limitations of this duality.

Example 1.2. Consider the flow on $\mathbf{R}^{3}$ given by

$$
(x, y, z) \cdot t=\left(x+a t, e^{b t} y, z+c t\right), \quad a, b, c>0,
$$

and let $\mathbf{Z} \oplus \mathbf{Z}$ act on $\mathbf{R}^{3}$ by $(x, y, z) \cdot(n, m)=\left(x+n,(-1)^{n} y, z+m\right)$. The flow and the group action commute, so there is a flow induced on $M=$ $\mathbf{R}^{3} / \mathbf{Z} \oplus \mathbf{Z}$. The group action is free, so $M$ is a 3-manifold. Its only isolated invariant set is

$$
S=(\mathbf{R} \times\{0\} \times \mathbf{R}) / \mathbf{Z} \oplus \mathbf{Z},
$$

which is a torus, and is a repeller in $M$. An index pair for $S$ in the forward flow is $\left(\left(\mathbf{R} \times D^{1} \times \mathbf{R}\right) / \mathbf{Z} \oplus \mathbf{Z},(\mathbf{R} \times\{-1,1\} \times \mathbf{R}) / \mathbf{Z} \oplus \mathbf{Z}\right)$; an index pair for the reverse flow is $((\mathbf{R} \times\{0\} \times \mathbf{R}) / \mathbf{Z} \oplus \mathbf{Z}, \varnothing)$. The homology indices are $C H_{*}^{+}(M ; S) \cong$ $\left(0, \mathbf{Z}_{2}, \mathbf{Z}_{2}, 0, \ldots\right), C H_{-}^{*}(M ; S) \cong(\mathbf{Z}, \mathbf{Z} \oplus \mathbf{Z}, \mathbf{Z}, 0, \ldots)$.

Example 1.3. Take flows on $S^{1}$ and $S^{2}$ so that each has a repelling fixed point. Form $X=S^{1} \vee S^{2}$ by wedging the two spheres together at the repellers. The wedge point is then an isolated invariant set in $X$, with index pair $\left(D^{1} \vee\right.$ $\left.D^{2}, S^{0} \vee S^{1}\right)$ in the forward flow and $\left(D^{1} \vee D^{2}, \varnothing\right)$ in the reverse flow. Thus $C H_{-}^{*}(X ; S)=(R, 0,0, \ldots), C H_{*}^{+}(X ; S)=(0, R \oplus R, R, 0, \ldots)$.

To establish a more general duality statement, manifolds with boundary will be considered. If $M$ is a manifold with boundary, its boundary $\partial M$ is closed and invariant under any flow on $M$. If $S$ is isolated in $M$ then $S \cap \partial M$ isolated in $\partial M$ [10]. An index quadruple supplies index pairs $\left(N_{0}, N_{0} \cap N_{1}\right)$ for $S \cap \partial M$ in $\partial M$ in the reverse flow, $\left(N_{0}, N_{0} \cap N_{2}\right)$ for $S \cap \partial M$ in $\partial M$ in the forward flow. Similarly, there is an index for $S$ in the pair $(M, \partial M)$, with $C H_{*}^{+}(M, \partial M ; S)$ for the forward flow are represented by $H_{*}\left(N, N_{0} \cup N_{2}\right)$; $C H_{*}^{-}(M, \partial M ; S)$ for the reverse flow represented by $H_{*}\left(N, N_{0} \cup N_{1}\right)$. The indices are related by the exact sequence

$$
\rightarrow C H_{*}^{ \pm}(\partial M, S \cap \partial M) \rightarrow C H_{*}^{ \pm}(M ; S) \rightarrow C H_{*}^{ \pm}(M, \partial M ; S) \rightarrow
$$


Example 1.4. Take the upper half-plane $\mathbf{H}^{2}$ with flow $\phi(x, y, t)=\left(e^{t} x, e^{t} y\right)$. The origin is the only isolated invariant set, with index quadruple $N=\{(x, y) \in$ $\left.\mathbf{H}^{+}: x^{2}+y^{2} \leq 1\right\}, N_{0}=\{(x, 0):|x| \leq 1\}, N_{1}=\varnothing, N_{2}=\left\{(x, y) \in \mathbf{H}^{+}: x^{2}+\right.$ $\left.y^{2}=1\right\}$. From these the homology indices are readily computed and seen to form the exact sequence above:

$$
\begin{array}{rlrl}
C H_{*}^{+}(\partial M ; S) & \cong(0, \mathbf{Z}, 0, \ldots), & C H_{*}^{-}(\partial M ; S) \cong(\mathbf{Z}, 0,0, \ldots), \\
C H_{*}^{+}(M ; S) & \cong(0,0,0, \ldots), \quad C H_{*}^{-}(M ; S) \cong(\mathbf{Z}, 0,0, \ldots), \\
C H_{*}^{+}(M, \partial M ; S) & \cong(0,0, \mathbf{Z}, 0, \ldots), \quad C H_{*}^{-}(M, \partial M ; S) \cong(0,0,0, \ldots) .
\end{array}
$$

It is sometimes possible to simplify the computation of index pairs by decomposing the isolated invariant set. The simplest such decomposition is to recognize $S$ as a sum or a product. If $S$ is the disjoint union of two isolated invariant sets $S_{1}$ and $S_{2}$, then the Conley index of $S$ is the wedge product of the indices of $S_{1}$ and $S_{2}: I(M ; S)=I\left(M ; S_{1}\right) \vee I\left(M ; S_{2}\right)$. The homology index is then additive: $C H_{*}^{ \pm}(M ; S)=C H_{*}^{ \pm}\left(M ; S_{1}\right) \oplus C H_{*}^{ \pm}\left(M ; S_{2}\right)$.

Similarly, if $M$ is a product $M_{1} \times M_{2}$ with the flow preserving the product structure and $S=S_{1} \times S_{2}$, then the Conley index of $S$ is the smash product of the indices of $S_{1}$ and $S_{2}: I(M ; S)=I\left(M_{1} ; S_{1}\right) \wedge I\left(M_{2} ; S_{2}\right)$. The homology index is given by the Künneth formula. Of course, in any manifold, if some neighborhood of $S$ can be coordinatized so that the flow has such a product structure, the same product formula applies. This can be clearly seen in Examples 1.1 and 1.2.

One of the key properties of the Conley index is its stability under perturbation of the flow. This stability, referred to as continuation, is described as follows. Let $M \times \Lambda$ be a parameterized family of flows, and let $M_{\lambda}$ represent $M$ with the $\lambda$-flow. Let $\mathscr{S}(M)=\left\{\left(S_{\lambda}, \lambda\right): S_{\lambda}\right.$ is isolated in $\left.M_{\lambda}\right\}$. For every compact $N \subseteq M$, the set $\Lambda(N)$ of parameter values for which $N$ is isolating is open. Topologize $\mathscr{S}(M)$ by taking as a subbasis sets of the form $\sigma_{N}(U)=\left\{\left(S_{\lambda}, \lambda\right): \lambda \in U, U \subseteq \Lambda(N)\right.$ open, $S_{\lambda}$ the maximal invariant set of $N$ in $\left.M_{\lambda}\right\}$. Then $\left(S_{\mu}, \mu\right)$ and $\left(S_{\lambda}, \lambda\right)$ are related by continuation if they lie in the same path component of $\mathscr{S}(M)$. That is, if there exists a collection of sets $K_{i} \subseteq \Lambda$ covering a path from $\lambda$ to $\mu$, and sets $N_{i} \subset M$ such that $K_{i} \subseteq \Lambda\left(N_{i}\right)$ and $S_{\lambda}=\sigma_{N_{1}}\left(K_{1}\right), S_{\mu}=\sigma_{N_{n}}\left(K_{n}\right)$.

The stability property of the Conley index is that if $\left(S_{\lambda}, \lambda\right)$ and $\left(S_{\mu}, \mu\right)$ are related by continuation, than $I\left(M_{\lambda} ; S_{\lambda}\right)$ and $I\left(M_{\mu} ; S_{\mu}\right)$ are isomorphic. This isomorphism is established by showing that the neighborhoods $K_{i}$ above can be chosen sufficiently small that for each $K$ and every $\kappa \in K$, the inclusion $\left(X_{\kappa}, S_{\kappa}\right) \rightarrow(X \times K, S(K))$ induces an index isomorphism. The composition isomorphism $F(\lambda, \mu): I\left(M_{\lambda} ; S_{\lambda}\right) \rightarrow I\left(M_{\mu} ; S_{\mu}\right)$ derived from these inclusions and their inverses depends only on the homotopy class of the path from $\lambda$ to $\mu$. For the homology index then, there is an isomorphism

$$
F_{*}(\lambda, \mu): C H_{*}^{+}\left(M_{\lambda} ; S_{\lambda}\right) \rightarrow C H_{*}^{+}\left(M_{\mu} ; S_{\mu}\right) .
$$

Example 1.5. In [2], the index is used to prove the existence and stability of magnetohydrodynamic shock waves. In the system studied, there are four rest points $u_{0}, u_{1}, u_{2}, u_{3}$, and the shock waves correspond to connecting orbits 
between these points. The points are all hyperbolic, with $u_{i}$ having index $\Sigma^{i}$. The main points of the argument developed in [2] are:

(i) The set of bounded solutions with positive volume and temperature $S$ is isolated.

(ii) The flow is gradient-like [1], so $S$ consists of the four rest points and connecting orbits between them. Thus if there are no connecting orbits, the homology index is the sum of the indices of the rest points $u_{i}: C H_{*}^{+}(M ; S) \cong$ $(\mathbf{Z}, \mathbf{Z}, \mathbf{Z}, \mathbf{Z}, 0, \ldots)$.

(iii) As the energy of the system is increased, the flow continues to one with no rest points, so $S$ is related by continuation to the empty set and $\mathrm{CH}_{*}^{+}(M ; S)=$ 0 . Thus connecting orbits (i.e. shock waves) exist. Further, these solutions are stable, as the continuation argument used is stable.

The Conley index arose as a generalization of Morse theory. Example 1.1 shows this: a nondegenerate critical point with Morse index $p$ is an isolated invariant set with Conley index $\Sigma^{p}$. The key elements of Morse theorydecomposing a manifold by unstable manifolds of critical points of a gradient flow, and relating the topology of the manifold to the numbers of critical points-are likewise extended in the Conley index theory. These generalizations are Morse decompositions and (generalized) Morse inequalities. Closely related is the algebraic machinery of attractor-repeller sequences and connection matrices.

If $(P,<)$ is a partially ordered indexing set, an interval in $P$ is a subset $I \subset P$ such that, if $p<q<r, p, r \in I$, then $q \in I$. An attracting interval is an interval $J$ such that, if $p \in J, q<p$, then $q \in J$. A collection of subinvariant sets (Morse sets) $\mathscr{S}=\left\{S_{p}\right\}_{p \in P}$ is a $(P,<)$-ordered Morse decomposition of $S$ in $M$ if each $S_{p}$ is isolated in $M$ and every orbit in $S \backslash\left(\bigcup_{p \in P} S_{p}\right)$ runs from $S_{q}$ to $S_{p}$ for some $p<q$.

Then for each interval $I \subseteq P$, the set $S(I)=\left\{x \in S: \omega(x) \subseteq S_{p}, \omega^{*}(p) \subseteq S_{q}\right.$ for some $p, q \in I\}$ is isolated in $M$. Index pairs for all of the isolated invariant sets $S(I)$ are produced simultaneously by an index filtration [6]: a collection $\{N(J): J$ attracting interval $\}$ such that $(N(J), N(\varnothing))$ is an index pair for $S(J)$ and $N\left(J_{1}\right) \cup N\left(J_{2}\right)=N\left(J_{1} \cup J_{2}\right), N\left(J_{1}\right) \cap N\left(J_{2}\right)=N\left(J_{1} \cap J_{2}\right)$. Then for any interval $I$, there exist attracting intervals $J, K$ so that $I=K \backslash J$, and for any such $J, K,(N(K), N(J))$ is an index pair for $S(I)$.

The simplest nontrivial case of a Morse decomposition is an attractor-repeller pair: $P=\{0,1\}$ with $0<1 ; S_{0}=A$ an attractor in $S ; S_{1}=A^{*}$ its dual repeller. In this case, the index filtration is a nested triple of compact spaces $N_{0} \subseteq N_{1} \subseteq N_{2}$. These may be chosen so that $N_{0}$ and $N_{1}$ are neighborhood retracts in $N_{2}$. Such a triple is called a regular index triple, and the exact sequence of the triple

$$
\stackrel{\partial}{\longrightarrow} H_{k}\left(N_{1}, N_{0}\right) \rightarrow H_{k}\left(N_{2}, N_{0}\right) \rightarrow H_{k}\left(N_{2}, N_{1}\right) \stackrel{\partial}{\longrightarrow}
$$

then defines the homology attractor-repeller sequence

$$
\stackrel{\partial}{\longrightarrow} C H_{k}(M ; A) \rightarrow C H_{k}(M ; S) \rightarrow C H_{k}\left(M ; A^{*}\right) \stackrel{\partial}{\longrightarrow} .
$$

For a general Morse decomposition $\mathscr{S}=\left\{S_{p}\right\}_{p \in P}$, a pair of intervals $(I, J)$ is said to be adjacent if $I J:=I \cup J$ is an interval and no $i \in I, j \in J$ has 
$j<i$. Every adjacent pair of intervals generates an index triple for the attractorrepeller decomposition $(S(I), S(J))$ of $S(I J)$, and so generates a homology attractor-repeller sequence. To understand how all of these induced homology sequences are related, we reformulate the sequence as follows:

In field coefficients, the sequence

$$
\stackrel{\partial}{\longrightarrow} C H_{k}(M ; S(I)) \rightarrow C H_{k}(M ; S(I J)) \rightarrow C H_{k}(M ; S(J)) \stackrel{\partial}{\longrightarrow}
$$

defines a matrix $\Delta=\left[\begin{array}{ll}0 & \partial \\ 0 & 0\end{array}\right]$ on the vector space $C H_{*}(M ; S(I)) \oplus C H_{*}(M ; S(J))$. As $\Delta^{2}=0,\left\{C H_{*}(M ; S(I)) \oplus C H_{*}(M ; S(J)), \Delta\right\}$ may be viewed as a chain complex. Further, if $C H_{*}(M ; S(I))$ and $C H_{*}(M ; S(J))$ are viewed as chain complexes with trivial boundary operators, then the sequence

$0 \rightarrow C H_{*}(M ; S(I)) \rightarrow C H_{*}(M ; S(I)) \oplus C H_{*}(M ; S(J)) \rightarrow C H_{*}(M ; S(J)) \rightarrow 0$

is a short exact sequence of chain complexes. Let $H_{*} \Delta(I J)$ denote the homology of $\left\{C H_{*}(M ; S(I)) \oplus C H_{*}(M ; S(J)), \Delta\right\}$. There is an isomorphism of homology sequences

$$
\begin{aligned}
& \stackrel{\partial}{\rightarrow} C H_{k}(M ; S(I)) \rightarrow \quad H_{k} \Delta(I J) \quad \rightarrow C H_{k}(M ; S(J)) \stackrel{\text { }}{\rightarrow} \\
& \text { id } \quad \cong \quad \quad \text { id } \\
& \stackrel{\partial}{\rightarrow} C H_{k}(M ; S(I)) \rightarrow C H_{k}(M ; S(I J)) \rightarrow C H_{k}(M ; S(J)) \stackrel{\stackrel{\leftrightarrow}{\rightarrow}}{ }
\end{aligned}
$$

so the matrix $\Delta$ computes the homology attractor-repeller sequence. Such a matrix is defined for every adjacent pair of intervals. In [7], Franzosa shows that all of these matrices and their induced homology sequences may be derived from a single matrix, known as a connection matrix.

To do so, fix a coefficient field for all homology groups. For every interval $J$, let $C_{*} \Delta(J)=\bigoplus_{p \in J} C H_{*}\left(M ; S_{p}\right)$. If $\Delta: C_{*} \Delta(P) \rightarrow C_{*} \Delta(P)$ is a matrix, $\Delta_{p, q}$ is a linear map from $C H_{*}\left(M ; S_{q}\right)$ to $C H_{*}\left(M ; S_{p}\right) . \Delta$ is said to be strictly upper triangular if $\Delta_{p, q}=0$ for all $p \nless q$; and is said to be a boundary map if each $\Delta_{p, q}$ is degree -1 and $\Delta^{2}=0$. If $\Delta: C_{*} \Delta(P) \rightarrow C_{*} \Delta(P)$ is a strictly upper triangular boundary map, $I, J$ intervals in $P$, define $\Delta(J, I): C_{*} \Delta(J) \rightarrow$ $C_{*} \Delta(I)$ by $\Delta(J, I)=\left[\Delta_{p, q}\right]_{p \in I, q \in J}$, and denote $\Delta(J, J)$ by $\Delta(J)$. It is easily computed that $\Delta(J)$ is a strictly upper triangular boundary map on $C_{*} \Delta(J)$.

That is, $\Delta$ defines a chain complex $\left\{C_{*} \Delta(J), \Delta(J)\right\}$ for every interval, whose homology is denoted by $H_{*} \Delta(J)$. Note that for $p \in P,\left\{C_{*} \Delta(p), \Delta(p)\right\}=$ $\left\{C H_{*}\left(M ; S_{p}\right), 0\right\}$ so $H_{*} \Delta(p)=C H_{*}\left(M ; S_{p}\right)$. Further, if $(I, J)$ is an adjacent pair of intervals, then the inclusions and projections of factors give a short exact sequence of chain complexes

$$
0 \rightarrow C_{*} \Delta(I) \rightarrow C_{*} \Delta(I J) \rightarrow C_{*} \Delta(J) \rightarrow 0
$$

and hence a long exact homology sequence

$$
\cdots \stackrel{\partial(J, I)}{\longrightarrow} H_{*} \Delta(I) \rightarrow H_{*} \Delta(I J) \rightarrow H_{*} \Delta(J) \stackrel{\partial(J, I)}{\longrightarrow} \cdots
$$

where $\partial(J, I)$ is the induced map on homology of $\Delta(J, I)$.

A connection matrix on $C_{*} \Delta(P)$ is a strictly upper triangular boundary map $\Delta: C_{*} \Delta(P) \rightarrow C_{*} \Delta(P)$ and a collection of maps $\{\phi(J): J$ an interval $\}$ with $\phi(J): H_{*} \Delta(J) \rightarrow C H_{*}(M ; S(J))$ such that $\phi(p)=$ id for all $p \in P$, and 
such that, for every adjacent pair of intervals $(I, J)$, the following diagram commutes:

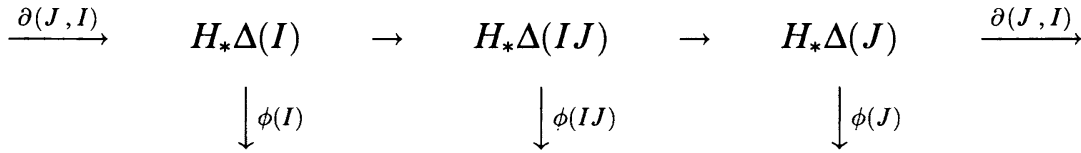

$$
\begin{aligned}
& \stackrel{\partial}{\longrightarrow} C H_{k}(M ; S(I)) \rightarrow C H_{k}(M ; S(I J)) \rightarrow C H_{k}(M ; S(J)) \stackrel{\partial}{\longrightarrow}
\end{aligned}
$$

A simple induction argument shows that all of the $\phi(J)$ 's are in fact isomorphisms. Thus a connection matrix computes (up to isomorphism) all homology attractor-repeller sequences. Connection matrices exist for all Morse decompositions [7], but are not unique. We will denote the set of connection matrices defined for $\mathscr{S}$ with ordering $(P,<)$ by $C M_{*}^{+}(\mathscr{S},<)$.

This construction is based on the homology index $\mathrm{CH}_{*}^{+}$. Similar constructions can be made using $\mathrm{CH}_{*}^{-}, \mathrm{CH}_{-}^{*}, \mathrm{CH}_{+}^{*}$ (connection matrices based on cohomology will be degree +1 coboundary maps). The set of connection matrices defined on $\mathrm{CH}_{*}^{-}$will be denoted $\mathrm{CM}_{*}^{-}(\mathscr{S},<)$; the set defined on $\mathrm{CH}_{-}^{*}$ is denoted $C M_{-}^{*}(\mathscr{S},<)$, etc.

The Morse inequalities, as formulated by Conley [4], follow naturally from the existence of connection matrices. Namely, given a finite chain complex $\{C, d\}$ with field coefficients, $\operatorname{rk}\left(C_{k}\right)=\operatorname{rk}\left(Z_{k}\right)+\operatorname{rk}\left(B_{k-1}\right)$ and $\operatorname{rk}\left(Z_{k}\right)=$ $\operatorname{rk}\left(H_{k}(C)\right)+\operatorname{rk}\left(B_{k}\right)$. Thus $\operatorname{rk}\left(C_{k}\right)=\operatorname{rk}\left(H_{k}(C)\right)+\operatorname{rk}\left(B_{k-1}\right)+\operatorname{rk}\left(B_{k}\right)$. For each of the graded groups $C_{*}, H_{*}(C), B_{*}$, there are Poincare polynomials $P\left(C_{*}, t\right)=\operatorname{rk}\left(C_{k}\right) t^{k}, P\left(H_{*}(C), t\right)=\operatorname{rk}\left(H_{k}(C)\right) t^{k}, P\left(B_{*}, t\right)=\operatorname{rk}\left(B_{k}\right) t^{k}$, which then satisfy the equation $P\left(C_{*}, t\right)=P\left(H_{*}(C)\right)+(1+t) P\left(B_{*}\right)$. Applying this now to the chain complex $\left\{C_{*} \Delta(P), \Delta(P)\right\}$ generated by a connection matrix yields the Morse inequalities:

$$
\sum_{p \in P} P\left(S_{p}, t\right)=P(S, t)+(1+t) P\left(\operatorname{im} \Delta_{*}(P), t\right)
$$

where $P(S, t)=P\left(C H_{*}(M ; S), t\right)$ and $P\left(S_{p}, t\right)=P\left(C H_{*}\left(M: S_{p}\right), t\right)$.

Consider for instance the system in Example 1.5. As the system is gradientlike, the four rest points form a Morse decomposition for $S$, with ordering $0<1<2<3$. Just as $S$ continues to the empty set for high enough energy, so too $S(01)$ and $S(23)$ continue to the empty set. The attractor-repeller sequences of $u_{0}$ and $u_{1}$ in $S(01)$, and of $u_{2}$ and $u_{3}$ in $S(23)$, can then be computed. In both cases, $C H_{*}(M ; S(I))$ is zero, so the connection map $\partial: C H_{p}\left(M ; u_{i}\right) \rightarrow C H_{p-1}\left(M ; u_{i-1}\right)$ is an isomorphism. Thus there exist connecting orbits from $u_{1}$ to $u_{0}$ and from $u_{3}$ to $u_{2}$. These maps also appear as entries in the connection matrix of the system, and are in fact enough to compute the entire matrix. Taking only the nonzero dimensions of each $C H_{*}\left(M ; u_{i}\right)$, any connection matrix is a $4 \times 4$ matrix with only the first upper diagonal nonzero:

$$
\Delta=\left[\begin{array}{llll}
0 & \partial_{01} & 0 & 0 \\
0 & 0 & \partial_{12} & 0 \\
0 & 0 & 0 & \partial_{23} \\
0 & 0 & 0 & 0
\end{array}\right]
$$

The attractor-repeller sequences of $S(01)$ and $S(12)$ show that $\partial_{01}$ and $\partial_{23}$ are nonzero, while the requirement $\Delta^{2}=0$ forces $\partial_{12}=0$. Thus the shock waves 
corresponding to the $u_{1}$-to- $u_{0}$ and $u_{3}$-to- $u_{2}$ connections exist for all parameter values, while connections from $u_{2}$ and $u_{1}$ are not guaranteed algebraically, and do not exist for all parameter values (cf. [8]).

In the Morse inequality description, $\sum_{p \in P} P\left(S_{p}, t\right)=1+t+t^{2}+t^{3}$, while $P(S, t)=0$, so $(1+t) P\left(\operatorname{im} \Delta_{*}(P), t\right)=1+t+t^{2}+t^{3}$ and $P\left(\operatorname{im} \Delta_{*}(P), t\right)=$ $1+t^{2}$.

\section{PoINCARÉ-Lefschetz DUAlity}

In Example 1.1, both the forward and reverse homology indices are suspensions of the homology of $S$, so the duality of the forward and reverse indices is trivial. We show now that duality holds quite independent of the topology of $S$ and the orientability of the fibrations

$$
\begin{aligned}
& \left(D^{s} \times D^{u}, D^{s} \times S^{u-1}\right) \rightarrow\left(N, N_{2}\right) \rightarrow S, \\
& \left(D^{s} \times D^{u}, S^{s-1} \times D^{u}\right) \rightarrow\left(N, N_{1}\right) \rightarrow S .
\end{aligned}
$$

However, as Examples 1.2 and 1.3 indicate, orientability of the ambient manifold and the possible intersection of the invariant set with the boundary of the manifold must be taken into account.

Theorem 2.1. If $M$ is an orientable n-manifold with boundary, $S \subseteq M$ an isolated invariant set for a $C^{1}$ flow on $M$, then there exist duality isomorphisms

$$
\begin{aligned}
& D: C H_{-}^{*}(M, \partial M ; S) \rightarrow C H_{*}^{+}(M ; S), \\
& D: C H_{-}^{*}(M ; S) \rightarrow C H_{*}^{+}(M, \partial M ; S) .
\end{aligned}
$$

Further, if $S \cap \partial M=\varnothing$, both isomorphisms reduce to an isomorphism

$$
D: C H_{-}^{*}(M ; S) \rightarrow C H_{*}^{+}(M ; S) \text {. }
$$

Proof. The proof consists of two steps, which are carried out in the lemmas below:

(i) For each quadruple $\left(N, N_{0}, N_{1}, N_{2}\right) \in \mathscr{M}(M, \partial M ; S)$, there exist isomorphisms $\cap z_{N}: H^{k}\left(N, N_{0} \cup N_{1}\right) \rightarrow H_{n-k}\left(N, N_{2}\right)$ and $\cap z_{N}: H^{k}\left(N, N_{1}\right)$ $\rightarrow H_{n-k}\left(N, N_{0} \cup N_{2}\right)$, where $z_{N} \in H_{n}(N, \partial N)$ is the fundamental class of $(N, \partial N)$.

(ii) The cap product isomorphisms commute with the bonding maps of the inverse system of index quadruples. Given $\left(N, N_{0}, N_{1}, N_{2}\right),\left(N^{\prime}, N_{0}^{\prime}, N_{1}^{\prime}, N_{2}^{\prime}\right)$ $\in \mathscr{M}(M, \partial M ; S)$ with $N^{\prime} \subseteq N$, the homotopy equivalences $\phi_{-}$and $\phi^{+}$induce a commutative diagram

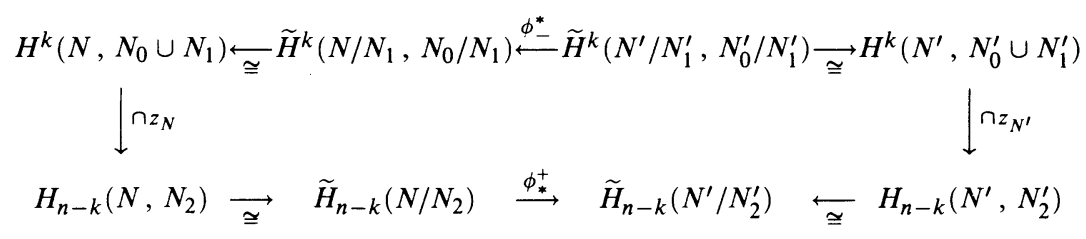

The isomorphism $D: C H_{-}^{*}(M, \partial M ; S) \rightarrow C H_{*}^{+}(M ; S)$ is then the inverse limit of the cap products $\cap z_{N}$.

Lemma 2.3. If $M$ is an orientable n-manifold, $S \subseteq M$ an isolated invariant 
set, and $\left(N, N_{0}, N_{1}, N_{2}\right) \in \mathscr{M}(M, \partial M ; S)$, then the maps

$$
\begin{aligned}
& \cap z_{N}: H^{k}\left(N, N_{0} \cup N_{1}\right) \rightarrow H_{n-k}\left(N, N_{2}\right), \\
& \cap z_{N}: H^{k}\left(N, N_{1}\right) \rightarrow H_{n-k}\left(N, N_{0} \cup N_{2}\right)
\end{aligned}
$$

are isomorphisms.

Proof. As the two isomorphisms are analogous, we prove only the first. Let $\tilde{N}$ be formed by identifying two copies of $N$ (denoted $N^{+}$and $N^{-}$) along $N_{2}$. For any subset $U$ of $N$, form $\widetilde{U} \subseteq \widetilde{N}$ similarly. Note that $\widetilde{N}_{0} \cup \widetilde{N}_{1}=\partial \widetilde{N}$, and that $N_{2} \cap\left(\widetilde{N}_{0} \cup \widetilde{N}_{1}\right)=\partial N_{2} . N$ is an orientable manifold with fundamental class $z_{N}$, so $N^{+}$and $N^{-}$are orientable with fundamental classes $z_{+}, z_{-}$. Thus $\tilde{N}$ is an orientable manifold, with fundamental class $\tilde{z}$ the image of $\left(z_{+}, z_{-}\right)$ under the composition

$$
H_{n}\left(N^{+}, \partial N^{+}\right) \oplus H_{n}\left(N^{-}, \partial N^{-}\right) \rightarrow H_{n}\left(\tilde{N}, N_{2} \cup \partial \tilde{N}\right) \leftarrow H_{n}(\tilde{N}, \partial \tilde{N}) .
$$

Further, as $N_{2}$ is a neat $n-1$ submanifold with boundary of $\tilde{N}$, with fundamental class $z_{2} \in H_{n-1}\left(N_{2}, \partial N_{2}\right)$ is the image of $\tilde{z}, z_{+}$and $z_{-}$under the appropriate boundary maps.

$N^{+}, N^{-}$and $N_{2}$ are all excisive in $\tilde{N}$, so there are exact sequences

$\rightarrow H^{k}(\tilde{N}, \partial \tilde{N}) \rightarrow H^{k}\left(N^{+}, N^{+} \cap \partial \tilde{N}\right) \oplus H^{k}\left(N^{-}, N^{-} \cap \partial \tilde{N}\right) \rightarrow H^{k}\left(N_{2}, \partial N_{2}\right) \rightarrow$ and

$$
\rightarrow H_{n-k}\left(N_{2}\right) \rightarrow H_{n-k}(\tilde{N}) \rightarrow H_{n-k}\left(N^{+}, N_{2}\right) \oplus H_{n-k}\left(N^{-}, N_{2}\right) \rightarrow .
$$

The cap products link the two sequences, forming the diagram:

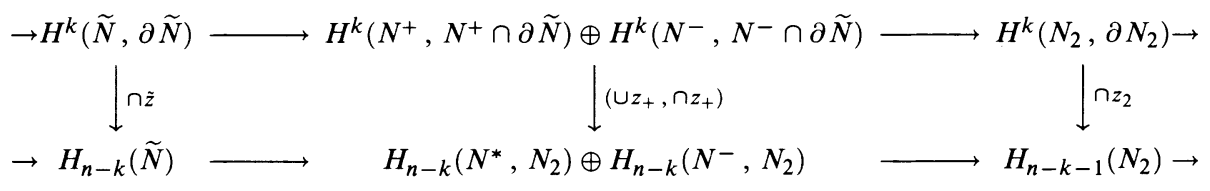

The cap product $\cap \tilde{z}$ commutes with $\left(\cap z_{+}, \cap z_{+}\right)$, and $\left(\cap z_{+}, \cap z_{+}\right)$commutes with $\cap z_{2}$, from the naturality of cap products and their commutativity with Mayer-Vietoris boundaries $[14,5.6 .16,5.6 .20]$. To show that $\cap \tilde{z}$ and $\cap z_{2}$ commute requires slightly more work. There are inclusion-induced diagrams

$$
\begin{aligned}
& H^{k}\left(N_{2}, \partial N_{2}\right) \stackrel{\cong}{\leftrightarrows} H^{k}\left(\partial N^{+}, N^{+} \cap \partial \tilde{N}\right) \longrightarrow H^{k}\left(\partial N^{+}\right) \\
& H_{n-k-1}\left(N_{2}\right) \longrightarrow H_{n-k-1}\left(\partial N^{+}\right) \stackrel{\text { id }}{\longleftarrow} H_{n-k-1}\left(\partial N^{+}\right)
\end{aligned}
$$

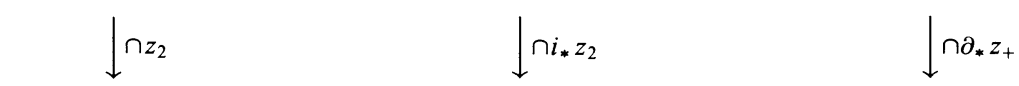

and

$$
\begin{aligned}
& H^{k+1}\left(N^{+}, \partial N^{+}\right) \longleftarrow H^{k+1}\left(\tilde{N}, N^{-} \cup \partial \tilde{N}\right) \longrightarrow H^{k+1}(\tilde{N}, \partial \tilde{N}) \\
& \downarrow \cap z_{+} \quad\left\lfloor\cap \tilde{z}_{+} \quad \downarrow \cap \tilde{z}\right. \\
& H_{n-k-1}\left(N^{+}\right) \longrightarrow H_{n-k-1}(\tilde{N}) \stackrel{\text { id }}{\longleftarrow} H_{n-k-1}(\tilde{N})
\end{aligned}
$$


which are linked by the commutative diagram

$$
\begin{array}{ccc}
H^{k}\left(\partial N^{+}\right) & \stackrel{\delta^{*}}{\longrightarrow} & H^{k+1}\left(N^{+}, \partial N^{+}\right) \\
\left\lfloor\cap \partial_{*} z\right. & & \downarrow \cap z_{+} \\
H_{n-k-1}\left(\partial N^{+}\right) & & H_{n-k-1}\left(N^{+}\right)
\end{array}
$$

The composition of $2.5,2.6$ and 2.7 produces the required commutative square.

By Poincaré-Lefschetz duality, the cap products $\cap z_{2}$ and $\cap \tilde{z}$ are isomorphisms, so $\cap z_{+}$and $\cap z_{-}$are isomorphisms. But each of these is just the cap product $\cap z_{N}: H^{k}\left(N, N_{0} \cup N_{1}\right) \rightarrow H_{n-k}\left(N, N_{2}\right)$.

This shows that each index quadruple induces a duality isomorphism. To show that all such isomorphisms are compatible, we first consider a special case of nested index pairs.

Lemma 2.8. Suppose $\left(N, N_{0}, N_{1}, N_{2}\right),\left(N^{\prime}, N_{0}^{\prime}, N_{1}^{\prime}, N_{2}^{\prime}\right) \in \mathscr{M}(M, \partial M ; S)$ with $N^{\prime} \subseteq \operatorname{int}_{M} N$ such that, if $x \cdot\left[t_{0}, t_{1}\right] \subseteq N$, and $x \cdot t_{0}, x \cdot t_{1} \in N^{\prime}$, then $x \cdot\left[t_{0}, t_{1}\right] \subseteq N^{\prime}$. Then diagram 2.2 commutes: for $c^{\prime} \in H^{k}\left(N^{\prime}, N_{0}^{\prime} \cup N_{1}^{\prime}\right)$,

$$
\left(\pi_{*}^{\prime}\right)^{-1} \circ \phi_{*} \circ \pi_{*}\left(\pi^{*} \circ \phi^{*} \circ\left(\pi^{\prime *}\right)^{-1}\left(c^{\prime}\right) \cap z_{N}\right)=c^{\prime} \cap z_{N^{\prime}} .
$$

Proof. Let $P^{-}=\left\{x \in N: \exists t<0\right.$ with $\left.x \cdot[t, 0] \subseteq N, x \cdot t \in N^{\prime}\right\}, P^{+}=$ $\left\{x \in N: \exists t>0\right.$ with $\left.x \cdot[0, t] \subseteq N, x \cdot t \in N^{\prime}\right\}$. Then $P^{+} \cap P^{-}=N^{\prime}$, and $N=P^{+} \cup P^{-} \cup\left\{x \in N: x \cdot\left[\tau_{+}(x), \tau_{-}(x)\right] \cap N^{\prime}=\varnothing\right\}$, where $x \cdot\left[\tau_{+}(x), \tau_{-}(x)\right]$ is the maximal orbit segment of $x$ contained in $N$. There are continuous maps $\theta^{-}: P^{-} \rightarrow \mathbf{R}, \theta^{+}: P^{+} \rightarrow \mathbf{R}$ defined by

$$
\begin{aligned}
& \theta^{-}(x)=\max \left\{t \leq 0: x \cdot[t, 0] \subseteq N, x \cdot t \in N^{\prime}\right\}, \\
& \theta^{+}(x)=\min \left\{t \geq 0: x \cdot[0, t] \subseteq N, x \cdot t \in N^{\prime}\right\}
\end{aligned}
$$

There is then a map $\phi: P^{+} \cup P^{-} \rightarrow N^{\prime}$ defined by

$$
\phi(x)= \begin{cases}x \cdot \theta^{+}(x), & x \in P^{+}, \\ x \cdot \theta^{-}(x), & x \in P^{-} .\end{cases}
$$

On $P^{+} \cap P^{-}=N^{\prime}, \theta^{+}=\theta^{-}=0$, so $\phi$ is well defined, and is the identity map when restricted to $N^{\prime}$. It maps $P^{-} \backslash N^{\prime}$ to $N_{2}^{\prime}, P^{+} \backslash N^{\prime}$ to $N_{1}^{\prime},\left(P^{+} \cup P^{-}\right) \cap$ $N_{0}$ to $N_{0}^{\prime}$, and $\partial_{N}\left(P^{+} \cup P^{-}\right)$to $N_{1}^{\prime} \cap N_{2}^{\prime}$. There is then a continuous map $\tilde{\phi}: N /\left(\left(N_{0} \cup N_{1}\right) \cap N_{2}\right) \rightarrow N^{\prime} /\left(\left(N_{0}^{\prime} \cup N_{1}^{\prime}\right) \cap N_{2}^{\prime}\right)$ defined by

$$
\tilde{\phi}(x)= \begin{cases}{[\phi(x)],} & x \in P^{+} \cup P^{-}, \\ {\left[N_{1}^{\prime} \cap N_{2}^{\prime}\right],} & \text { otherwise }\end{cases}
$$

Let $\pi: N \rightarrow N /\left(\left(N_{0} \cup N_{1}\right) \cap N_{2}\right)$ and $\pi^{\prime}: N^{\prime} \rightarrow N^{\prime} /\left(\left(N_{0}^{\prime} \cup N_{1}^{\prime}\right) \cap N_{2}^{\prime}\right)$ be the natural projections. Then the following diagram commutes:

$$
\begin{gathered}
H_{n}(N, \partial N) \longrightarrow H_{n}\left(N, N \backslash \text { int } N^{\prime}\right) \longleftrightarrow H_{n}\left(N^{\prime}, \partial N^{\prime}\right) \\
\cong \downarrow \pi_{*} \\
\cong \downarrow \pi_{*}^{\prime} \\
H_{n}\left(N /\left(N_{0} \cup N_{1}\right) \cap N_{2}, \longrightarrow \tilde{\phi}_{*}\left(\begin{array}{c}
H_{n}\left(N^{\prime} /\left(N_{0}^{\prime} \cup N_{1}^{\prime}\right) \cap N_{2}^{\prime},\right. \\
\left.\partial N^{\prime} /\left(N_{0}^{\prime} \cup N_{1}^{\prime}\right) \cap N_{2}^{\prime}\right) .
\end{array}\right.\right.
\end{gathered}
$$


Thus $\left(\pi_{*}^{\prime}\right)^{-1} \circ \tilde{\phi}_{*} \circ \pi_{*}(z)=z^{\prime}$, and the maps $\pi, \tilde{\phi}$ and $\pi^{\prime}$ induce a commutive diagram of cap products

$$
\begin{aligned}
& H^{k}\left(N, N_{0} \cup N_{1}\right) \stackrel{\pi^{*} \circ \tilde{\phi}^{*} \circ\left(\pi^{\prime *}\right)^{-1}}{\longleftarrow} H^{k}\left(N^{\prime}, N_{0}^{\prime} \cup N_{1}^{\prime}\right) \\
& \left\lfloor\cap z \quad \downarrow \cap z^{\prime}\right. \\
& H_{n-k}\left(N, N_{2}\right) \stackrel{\left(\pi_{*}^{\prime}\right)^{-1} \circ \tilde{\phi}_{*} \circ \pi_{*}}{\longrightarrow} H_{n-k}\left(N^{\prime}, N_{2}^{\prime}\right)
\end{aligned}
$$

To complete the proof, it suffices to show that $\phi_{*}^{+}=\left(\pi_{*}^{\prime}\right)^{-1} \circ \tilde{\phi}_{*} \circ \pi_{*}$ and that $\phi_{-}^{*}=\pi^{*} \circ \tilde{\phi}^{*} \circ\left(\pi^{\prime *}\right)^{-1}$. To do so, we show that the compositions

$$
\left(N /\left(\left(N_{0} \cup N_{1}\right) \cap N_{2}\right), N_{2} /\left(\left(N_{0} \cup N_{1}\right) \cap N_{2}\right)\right) \stackrel{\pi}{\longrightarrow}\left(N / N_{2}, *\right) \stackrel{\phi}{\longrightarrow}\left(N^{\prime} / N_{2}^{\prime}, *\right)
$$

and

$$
\begin{aligned}
& \left(N /\left(N_{0} \cup N_{1}\right) \cap N_{2}, N_{2} /\left(N_{0} \cup N_{1}\right) \cap N_{2}\right) \\
& \quad \stackrel{\tilde{\phi}}{\longrightarrow}\left(N^{\prime} /\left(N_{0}^{\prime} \cup N_{1}^{\prime}\right) \cap N_{2}^{\prime}, N_{2}^{\prime} /\left(N_{0}^{\prime} \cup N_{1}^{\prime}\right) \cap N_{2}^{\prime}\right) \stackrel{\pi}{\longrightarrow}\left(N^{\prime} / N_{2}^{\prime}, *\right)
\end{aligned}
$$

are homotopic. Define $\Phi:\left(N /\left(N_{0} \cup N_{1}\right) \cap N_{2}\right) \times[0,1] \rightarrow N^{\prime} / N_{2}^{\prime}$ by

$$
\begin{aligned}
& \Phi([x], s) \\
& = \begin{cases}{\left[x \cdot\left(3 s T+(1-s) \theta^{+}(x)\right)\right],} & x \in P^{+}, \\
& x \cdot\left[0,3 s T+(1-s) \theta^{+}(x)\right] \subseteq N, \\
{\left[N_{2}^{\prime}\right],} & x \cdot\left((1-s) \theta^{+}(x)\right) \cdot[s T, 3 s T] \subseteq N^{\prime},\end{cases} \\
& \text { otherwise. }
\end{aligned}
$$

As every $x$ with $x \cdot\left[t_{0}, t_{1}\right] \subseteq N$ and $x \cdot t_{0}, x \cdot t_{1} \in N^{\prime}$ has $x \cdot\left[t_{0}, t_{1}\right] \subseteq N^{\prime}$, the set $\left\{x \cdot\left[0,3 s T+(1-s) \theta^{+}(x)\right] \subseteq N, x \cdot\left((1-s) \theta^{+}(x)\right) \cdot[s T, 3 s T] \subseteq N^{\prime}\right\}$ has its boundary contained in $\left\{x: x \cdot\left(3 s T+(1-s) \theta^{+}(x)\right) \in N_{2}^{\prime}\right\}$, and so is mapped to $\left[N_{2}^{\prime}\right]$. Thus $\Phi$ is continuous, with $\Phi_{0}=\pi \circ \tilde{\phi}, \Phi_{1}=\phi^{+} \circ \pi$. There is a similar homotopy between $\pi \circ \tilde{\phi}$ and $\phi^{-} \circ \pi$.

Lemma 2.9. If $\left(N, N_{0}, N_{1}, N_{2}\right)$, $\left(N^{\prime}, N_{0}^{\prime}, N_{1}^{\prime}, N_{2}^{\prime}\right) \in \mathscr{M}(M, \partial M ; S)$ with $N^{\prime} \subseteq N$, then the diagram (2.2) commutes.

Proof. If $\left(N, N_{0}, N_{1}, N_{2}\right) \in \mathscr{M}(M, \partial M ; S), T>0$, let $N^{T}=\{x \in N: x \cdot$ $[-T, T] \subseteq N\}$, and let $N_{0}^{T}=N_{0} \cap N^{T}, N_{1}^{T}=\left(N_{1} \cdot T\right) \cap N^{T}, N_{2}^{T}=$ $\left(N_{2} \cdot(-T)\right) \cap N^{T}$. Then $\left(N^{T}, N_{0}^{T}, N_{1}^{T}, N_{2}^{T}\right) \in \mathscr{M}(M, \partial M ; S)$. Further, if $\left(N^{\prime}, N_{0}^{\prime}, N_{1}^{\prime}, N_{2}^{\prime}\right) \in \mathscr{M}(M, \partial M ; S)$, there is a $T$ such that $N^{T} \subseteq \operatorname{int}\left(N^{\prime}\right)$. Then $N^{\prime T} \subseteq N^{T}$, and each of the containments $N^{\prime T} \subseteq N^{\prime}, N^{T} \subseteq N, N^{\prime T} \subseteq$ $N^{T}$ satisfies the hypothesis of Lemma 2.8. Thus all of the maps $\phi^{+}, \phi_{-}$are defined, and the corresponding diagram commutes for each of these containments. Further, the maps $\phi^{+}$and $\phi_{-}$form commutative diagrams

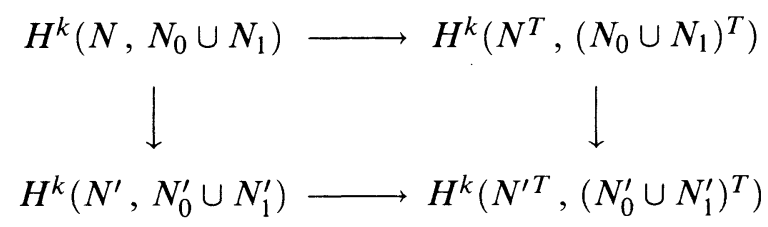


and

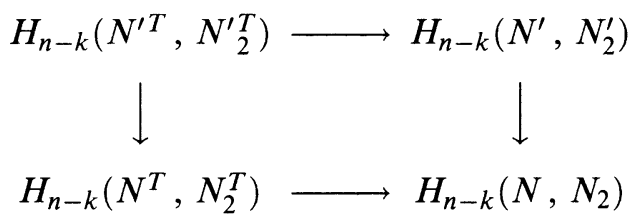

The composition of these then gives the required diagram.

If $S \cap \partial M=\varnothing$, then $C H_{-}^{p}(\partial M ; S \cap \partial M), C H_{p}^{+}(\partial M ; S \cap \partial M)=0$ and the indices of $S$ in $M$ and in $(M, \partial M)$ are isomorphic, via the exact sequences

$$
\begin{aligned}
& \rightarrow C H_{-}^{p}(M, \partial M ; S) \rightarrow C H_{-}^{p}(M: S) \rightarrow C H_{-}^{p}(\partial M ; S \cap \partial M) \rightarrow, \\
& \rightarrow C H_{p}^{+}(\partial M ; S \cap \partial M) \rightarrow C H_{p}^{+}(M ; S) \rightarrow C H_{p}^{+}(M, \partial M ; S) \rightarrow .
\end{aligned}
$$

There is then an isomorphism $D: C H_{-}^{*}(M ; S) \rightarrow C H_{*}^{+}(M ; S)$. Further, since $\partial(\partial M)=\varnothing, C H_{-}^{*}(\partial M ; S \cap \partial M)$ and $C H_{*}^{+}(\partial M ; S \cap \partial M)$ are dual. The exact sequences connect these various duality isomorphisms.

Theorem 2.10. If $S$ is isolated in $M$, the following diagram commutes:

$$
\begin{aligned}
& \rightarrow C H_{-}^{p}(M, \partial M ; S) \rightarrow C H_{-}^{p}(M ; S) \quad \rightarrow \quad C H_{p}^{p}(\partial M ; S \cap \partial M) \rightarrow \\
& \rightarrow C H_{n-p}^{+}(M ; S) \rightarrow C H_{n-p}(M, \partial M ; S) \rightarrow C H_{n-p-1}^{+}(\partial M ; S \cap \partial M) \rightarrow
\end{aligned}
$$

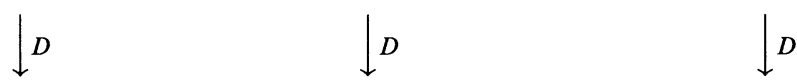

Proof. The sequences are represented by the exact sequences of the triples $\left(N, N_{0} \cup N_{1}, N_{1}\right)$ and $\left(N, N_{0} \cup N_{2}, N_{2}\right)$. If $z_{0} \in H_{n-1}\left(N_{0}, \partial N_{0}\right)$ is the fundamental class of $N_{0}, z_{0}$ is the image of $z$ under the Mayer-Vietoris boundary. Then the naturality of the cap product yields commutative diagram

$$
\begin{aligned}
& \rightarrow H^{k}\left(N, N_{0} \cup N_{1}\right) \rightarrow H^{k}\left(N, N_{1}\right) \quad \rightarrow \quad H^{k}\left(N_{0}, N_{0} \cap N_{1}\right) \quad \rightarrow \\
& \downarrow \cap z \quad \downarrow \cap z \quad \downarrow \cap z_{0} \\
& \rightarrow H_{n-k}\left(N, N_{2}\right) \rightarrow H_{n-k}\left(N, N_{0} \cup N_{2}\right) \rightarrow H_{n-k-1}\left(N_{0}, N_{0} \cap N_{2}\right) \rightarrow
\end{aligned}
$$

The index diagram is then the inverse limit of such diagrams.

\section{NATURALity}

Duality does more than just show that the homology indices are isomorphic as (ungraded) groups-it shows that for many of the constructions used in the Conley index theory, they carry the same information. To establish this, we show that the duality isomorphism is natural with respect to the basic properties of the Conley index: sums and products; continuation; attractor-repeller sequences and connection matrices. Examples show that other properties, such as ring structures and naturality with respect to semiconjugacies, are not preserved by duality.

Theorem 3.1. The duality isomorphism is natural with respect to sums and products of isolated invariant sets. That is, suppose $M$ and $M^{\prime}$ are manifolds with flows, $S \subseteq M$ and $S^{\prime} \subseteq M^{\prime}$ isolated invariant sets. Then 
(i) If $S_{1}, S_{2} \subseteq M$ are isolated so that $S_{1} \cup S_{2}=S, S_{1} \cap S_{2}=\varnothing$, then the following diagram commutes:

$$
\begin{aligned}
& C H_{-}^{k}\left(M, \partial M ; S_{1}\right) \oplus C H_{-}^{k}\left(M, \partial M ; S_{2}\right) \longleftarrow C H^{k}(M, \partial M ; S) \\
& \downarrow D_{1} \oplus D_{2} \\
& C H_{n-k}^{+}\left(M ; S_{1}\right) \oplus C H_{n-k}^{+}\left(M ; S_{2}\right) \quad \longrightarrow C H_{n-k}^{+}(M ; S)
\end{aligned}
$$

(ii) The following diagram commutes:

$C H_{-}^{k}(M, \partial M ; S) \oplus C H_{-}^{k^{\prime}}\left(M^{\prime}, \partial M^{\prime} ; S^{\prime}\right) \rightarrow C H_{-}^{k+k^{\prime}}\left(M \times M^{\prime}, \partial\left(M \times M^{\prime}\right) ; S\right)$

$$
\downarrow D_{1} \oplus D_{2} \quad \downarrow D
$$

$$
C H_{n-k}^{+}(M ; S) \oplus C H_{n^{\prime}-k^{\prime}}^{+}\left(M^{\prime} ; S^{\prime}\right) \rightarrow C H_{n+n^{\prime}-k-k^{\prime}}^{+}\left(M \times M^{\prime}, S \times S^{\prime}\right)
$$

Proof. (i) Choose $\left(N, N_{0}, N_{1}, N_{2}\right) \in M\left(M, \partial M ; S_{1}\right),\left(N^{\prime}, N_{0}^{\prime}, N_{1}^{\prime}, N_{2}^{\prime}\right) \in$ $\mathscr{M}\left(M, \partial M ; S_{2}\right)$ such that $N \cap N^{\prime}=\varnothing$. Then $\left(N \cup N^{\prime}, N_{0} \cup N_{0}^{\prime}, N_{1} \cup N_{1}^{\prime}, N_{2} \cup\right.$ $\left.N_{2}^{\prime}\right) \in \mathscr{M}(M, \partial M ; S)$, and there is commutative diagram

$$
\begin{aligned}
& H^{k}\left(N, N_{0} \cup N_{1}\right) \oplus H^{k}\left(N^{\prime}, N_{0}^{\prime} \cup N_{1}^{\prime}\right) \longleftarrow H^{k}\left(N \cup N^{\prime}, N_{0} \cup N_{0}^{\prime} \cup N_{1} \cup N_{1}^{\prime}\right) \\
& \downarrow\left(\cap z_{1}\right) \oplus\left(\cap z_{2}\right) \quad \downarrow \cap z \\
& H_{n-k}\left(N, N_{2}\right) \oplus H_{n-k}\left(N^{\prime}, N_{2}^{\prime}\right) \quad \longrightarrow \quad H_{n-k}\left(N \cup N^{\prime}, N_{2} \cup N_{2}^{\prime}\right)
\end{aligned}
$$

(ii) Similarly, choose $\left(N, N_{0}, N_{1}, N_{2}\right) \in \mathscr{M}(M, \partial M ; S),\left(N^{\prime}, N_{0}^{\prime}, N_{1}^{\prime}, N_{2}^{\prime}\right)$ $\in \mathscr{M}\left(M^{\prime}, \partial M^{\prime} ; S^{\prime}\right)$. Then $\left(N \times N^{\prime},\left(N \times N_{0}^{\prime}\right) \cup\left(N_{0} \times N^{\prime}\right),\left(N \times N_{1}^{\prime}\right) \cup\right.$ $\left.\left(N_{1} \times N^{\prime}\right),\left(N \times N_{2}^{\prime}\right) \cup\left(N_{2} \times N^{\prime}\right)\right) \in \mathscr{M}\left(M \times M^{\prime}, \partial\left(M \times M^{\prime}\right) ; S \times S^{\prime}\right)$, and there is commutative diagram

$$
\begin{gathered}
H^{k}\left(N, N_{0} \cup N_{1}\right) \oplus H^{k^{\prime}}\left(N^{\prime}, N_{0}^{\prime} \cup N_{1}^{\prime}\right) \stackrel{\times}{\rightarrow} H^{k+k^{\prime}}\left(N \times N^{\prime},\left(N \times\left(N_{0}^{\prime} \cup N_{1}^{\prime}\right)\right) \cup\left(\left(N_{0} \cup N_{1}\right) \times N^{\prime}\right)\right) \\
\downarrow \\
D_{1} \oplus D_{2} \\
H_{n-k}\left(N, N_{2}\right) \oplus H_{n^{\prime}-k^{\prime}}\left(N^{\prime}, N_{2}^{\prime}\right) \stackrel{\times}{\rightarrow} H_{n+n^{\prime}-k-k^{\prime}}\left(N \times N^{\prime},\left(N \times N_{0}^{\prime}\right) \cup\left(N_{0} \times N^{\prime}\right)\right) \quad \square
\end{gathered}
$$

Theorem 3.2. The duality isomorphism is preserved under continuation. That is, if $M \times \Lambda$ is a parameterized family of flows with $\left(S_{\mu}, \mu\right)$ and $\left(S_{\lambda}, \lambda\right)$ related by continuation, then there exists commutative diagram of isomorphisms:

$$
\begin{array}{cc}
\qquad H_{-}^{p}\left(M_{\mu}, \partial M_{\mu} ; S_{\mu}\right) \underset{F^{*}(\lambda, \mu)}{\longrightarrow} C H_{-}^{p}\left(M_{\lambda}, \partial M_{\lambda} ; S_{\lambda}\right) \\
\qquad H_{n-p}^{+}\left(M_{\mu} ; S_{\mu}\right) & \underset{F_{*}(\mu, \lambda)}{\longrightarrow} C H_{n-p}^{+}\left(M_{\lambda} ; S_{\lambda}\right)
\end{array}
$$

Proof. Without loss, we may assume $\Lambda=[0,1]$ and that for every $t \in[0,1]$ $\left(X_{t}, S_{t}\right) \rightarrow(X \times \Lambda, S(\Lambda))$ is an index isomorphism. For any $U \subseteq X \times \Lambda, t \in \Lambda$, let $U_{t}=U \cap(X \times\{t\})$. Choose an index quadruple $\left(N(\Lambda), N_{0}(\Lambda), N_{1}(\Lambda)\right.$, $\left.N_{2}(\Lambda)\right)$ for $S(\Lambda)$. Then $\left(N_{t}, N_{0 t} \cup N_{1 t}\right)$ is an index pair for $\left(X_{t}, \partial X_{t} ; S_{t}\right)$ in the reverse flow; $\left(N_{t}, N_{2 t}\right)$ is an index pair for $\left(X_{t} ; S_{t}\right)$ in the forward flow. The inclusions $\left(N_{0}, N_{00} \cup N_{10}, N_{20}\right) \rightarrow\left(N, N_{0} \cup N_{1}, N_{2}\right)$ and $\left(N_{1}, N_{01} \cup\right.$ 
$\left.N_{11}, N_{21}\right) \rightarrow\left(N, N_{0} \cup N_{1}, N_{2}\right)$ induce diagram

$$
\begin{aligned}
& H^{k}\left(N_{0}, N_{00} \cup N_{10}\right) \stackrel{\cong}{\leftrightarrows} H^{k}\left(N, N_{0} \cup N_{1}\right) \stackrel{\cong}{\longmapsto} H^{k}\left(N_{1}, N_{01} \cup N_{11}\right) \\
& \downarrow \cap z_{0} n \quad \downarrow \cap z_{\Lambda} \quad \downarrow n z_{1} \\
& H_{n-k}\left(N_{0}, N_{20}\right) \stackrel{\cong}{\longrightarrow} H_{n-k}\left(N, N_{2}\right) \stackrel{\cong}{\longleftarrow} H_{n-k}\left(N_{1}, N_{21}\right)
\end{aligned}
$$

where the inclusion $H_{n}(X, \partial X) \stackrel{\cong}{\longrightarrow} H_{n}(X \times \Lambda, \partial X \times \Lambda)$ maps $z_{N}$ to $z_{\Lambda}$. But the horizontal compositions define the isomorphisms $F_{*}(0,1)$ and $F^{*}(1,0)$.

Theorem 3.3. The duality isomorphism commutes with attractor-repeller sequences. If $S$ is an isolated invariant set with attractor-repeller pair $\left(A, A^{*}\right)$, then the following diagrams commute:

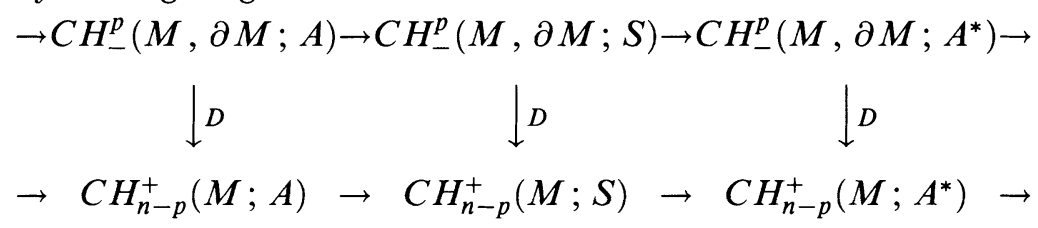

and

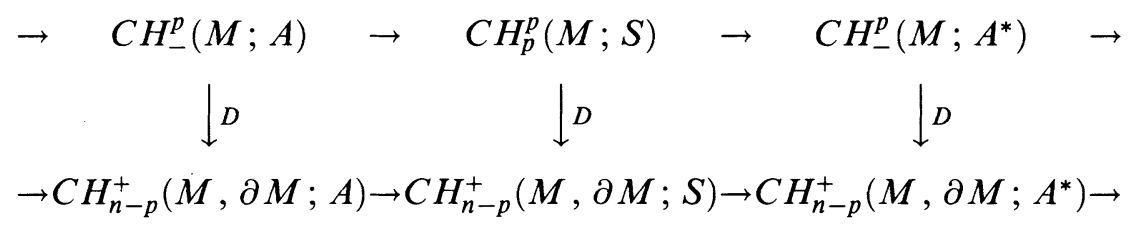

Proof. We show that the first diagram commutes. Choose $\left(N, N_{0}, N_{1}, N_{2}\right) \in$ $\mathscr{M}(M, \partial M ; S)$. There exist neighborhoods $L_{0}$ of $A$ and $L_{1}$ of $A^{*}$ in $N$ such that $L_{0} \cup L_{1}=N, \partial_{N} L_{0}=L_{0} \cap L_{1}=\partial_{N} L_{1}$, and such that $L_{0} \cap L_{1}$ lies in the exit set of $L_{1}$ and the entrance set of $L_{0}$ [12]. Then

$$
\begin{aligned}
& \left(L_{0}, N_{0} \cap L_{0},\left(N_{1} \cup L_{1}\right) \cap L_{0}, N_{2} \cap L_{0}\right) \in \mathscr{M}(M, \partial M ; A), \\
& \left(L_{1}, N_{0} \cap L_{1}, N_{1} \cap L_{1},\left(N_{2} \cup L_{0}\right) \cap L_{1}\right) \in \mathscr{M}\left(M, \partial M ; A^{*}\right) .
\end{aligned}
$$

Fundamental classes for $N, L_{0}$ and $L_{1}$ are related by the diagram

$$
\begin{gathered}
H_{n}\left(N, L_{1} \cup \partial N\right) \longleftarrow H_{n}(N, \partial N) \longrightarrow H_{n}\left(N, L_{0} \cup \partial N\right) \\
\uparrow \\
H_{n}\left(L_{0}, \partial L_{0}\right)
\end{gathered}
$$

The cap products of $N, L_{0}$ and $L_{1}$ link the exact sequences, forming diagram

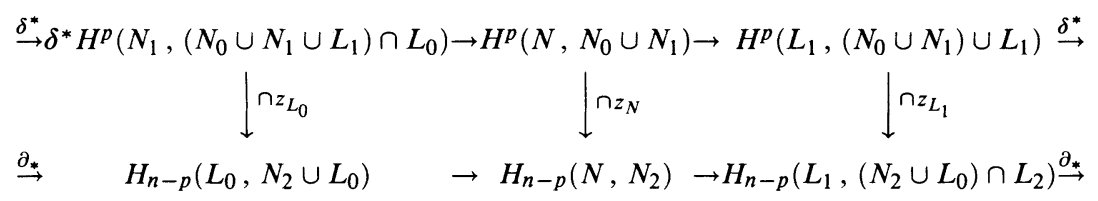

which represents the index diagram. 
The commutativity of $z_{L_{0}}$ and $z_{N}$, and of $z_{N}$ and $z_{L_{1}}$ follows from the naturality of the cap product. The commutativity of $z_{L_{1}}$ and $z_{L_{0}}$ is obtained by applying [5, VII, 12.25] with $X_{1}=L_{1} \cup N_{0} N_{1}, X_{2}=L_{0} \cup N_{2}, A_{1}=N_{0} \cup N_{1}$, $A_{2}=N_{2}$, and composing it with the diagrams induced by the inclusions

$$
\begin{aligned}
\left(L_{1},\right. & \left.\left(N_{0} \cup N_{1}\right) \cap L_{1},\left(N_{2} \cup L_{0}\right) \cap L_{1}\right) \\
& \rightarrow\left(L_{1} \cup N_{0} \cup N_{1}, N_{0} \cup N_{1},\left(\left(N_{0} \cup N_{1}\right) \cap L_{0}\right) \cup\left(\left(N_{2} \cup L_{0}\right) \cap L_{1}\right)\right.
\end{aligned}
$$

and

$$
\begin{aligned}
\left(L_{0},\right. & \left.\left(L_{1} \cup N_{0} \cup N_{1}\right) \cap L_{0}, N_{2} \cap L_{0}\right) \\
\quad & \rightarrow\left(L_{0} \cup N_{2},\left(\left(N_{0} \cup N_{1}\right) \cap L_{0}\right) \cup\left(\left(N_{2} \cup L_{0}\right) \cap L_{1}\right), N_{2}\right) .
\end{aligned}
$$

The composition yields the commutative diagram

$$
\begin{array}{ccc}
H^{p}\left(L_{1},\left(N_{0} \cup N_{1}\right) \cap L_{1}\right) & \stackrel{\delta^{*}}{\longrightarrow} H^{p+1}\left(L_{0},\left(N_{0} \cup N_{1} \cup L_{1}\right) \cap L_{0}\right) \\
\downarrow & & \left\lfloor\cap z_{L_{0}}\right. \\
H_{n-p}\left(L_{1},\left(N_{2} \cup L_{0}\right) \cap L_{1}\right) \stackrel{\partial_{*}}{\longrightarrow} & H_{n-p-1}\left(L_{0}, N_{2} \cap L_{0}\right) . & \square
\end{array}
$$

Note that if field coefficients $F$ are used, there is a natural isomorphism $C H_{ \pm}^{*}(M ; S) \rightarrow \operatorname{Hom}\left(C H_{*}^{ \pm}(M ; S), F\right)$, and a (noncanonical) isomorphism $C H_{*}^{ \pm}(M ; S) \cong \operatorname{Hom}\left(C H_{*}^{ \pm}(M ; S), F\right)$. That is, if $S \cap \partial M=\varnothing$, then the four attractor-repeller sequences (i.e. those of $\mathrm{CH}_{*}^{ \pm}$and $C H_{ \pm}^{*}$ ) all express the same information.

In a Morse decomposition $\mathscr{S}=\left\{S_{p}\right\}$ of $S$ (assume now that $S \cap \partial M=\varnothing$ ), $C H_{-}^{*}\left(M ; S_{p}\right)$ and $C H_{*}^{+}\left(M ; S_{p}\right)$ are dual for each $p$, with duality isomorphism $D_{p}$. Thus $C_{-}^{*} \Delta(P)=\bigoplus_{p \in P} C H_{-}^{*}\left(M ; S_{p}\right)$ and $C_{*}^{+} \Delta(P)=\bigoplus_{p \in P} C H_{*}^{+}\left(M ; S_{p}\right)$ are dual, with duality isomorphism $D=\bigoplus_{p \in P} D_{p}$.

Theorem 3.4. There is a bijection $\widetilde{D}: C M_{-}^{*}(\mathscr{S},<) \rightarrow C M_{*}^{+}(\mathscr{S},<)$, with $\widetilde{D}\left(\left\{\Delta_{-}^{*}(P), \phi_{-}^{*}(I)\right\}\right)=\left\{D \circ \Delta_{-}^{*}(P) \circ D^{-1}, D(I) \circ \phi_{-}^{*}(I) \circ D(I)^{-1}\right\}$.

Proof. It suffices to show that $\widetilde{D}$ does indeed map into $C M_{*}^{+}(\mathscr{S},<)$. If it does, then it has an inverse $\widetilde{D}^{-1}$ defined analogously from $D^{-1}$.

(i) $\Delta_{*}^{+}(P)$ is the composition

$$
C \Delta_{k}^{+}(P) \stackrel{D^{-1}}{\longrightarrow} C \Delta_{-}^{n-k}(P) \stackrel{\Delta}{\longrightarrow} C \Delta_{-}^{n-k+1}(P) \stackrel{D}{\longrightarrow} C \Delta_{k-1}^{+}(P),
$$

so it is a degree -1 map with $\Delta_{p, q}^{+}=D \circ 0 \circ D^{-1}=0$ for $p \nless q$ and with

$\Delta_{*}^{+}(P) \circ \Delta_{*}^{+}(P)=D \circ \Delta_{+}^{*}(P) \circ D^{-1} \circ D \circ \Delta_{+}^{*}(P) \circ D^{-1}=D \circ \Delta_{+}^{*}(P) \circ \Delta_{+}^{*}(P) \circ D^{-1}=0$.

That is, it is a strictly upper triangular boundary map.

For any adjacent pair of intervals $(I, J)$ (with respect to the forward flow, so that $(J, I)$ is an adjacent interval in the reverse flow), there is then an isomorphism of short exact chain complex sequences

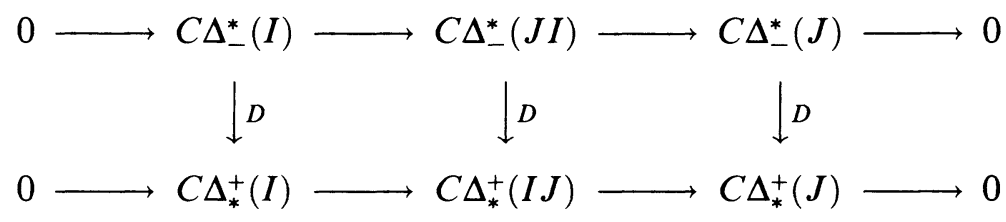


which induces an isomorphism of long exact sequences

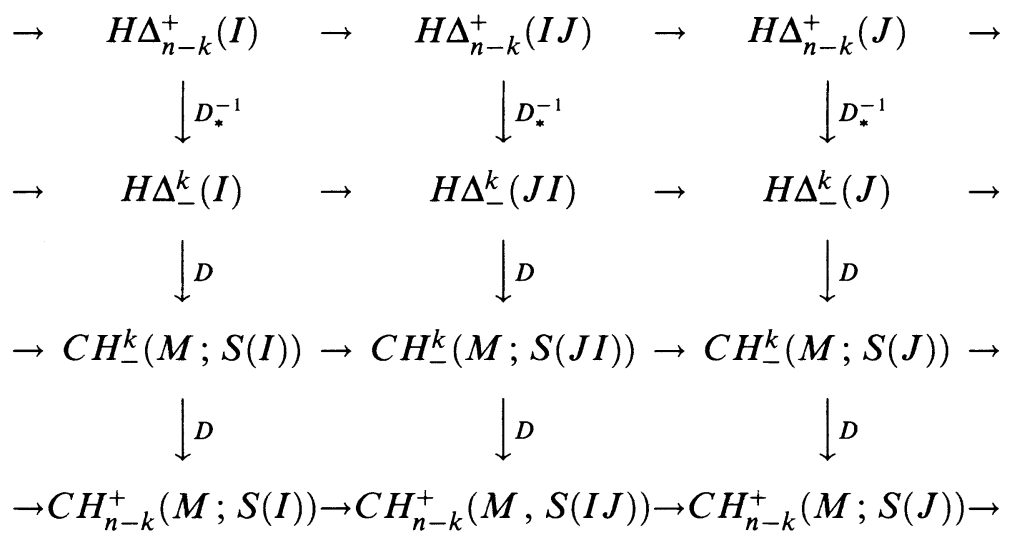

Thus the maps $D_{*}^{-1} \circ \phi_{+}(I) \circ D$ make the diagram commute, with $D_{*}^{-1} \circ \phi_{+}(p) \circ$ $D=D^{-1} \circ$ id $\circ D=\mathrm{id}$, as required.

Duality divides the four collections of connection matrices into two pairs: $C M_{-}^{*}(\mathscr{S},<) \cong C M_{*}^{+}(\mathscr{S},<) ; C M_{+}^{*}(\mathscr{S},<) \cong C M_{*}^{-}(\mathscr{S},<)$. As with attractorrepeller sequences, these are further related by the Hom functor. There are bijections $B^{+}: C M_{*}^{+}(\mathscr{S},<) \rightarrow C M_{+}^{*}(\mathscr{S},<), B^{-}: C M_{*}^{-}(\mathscr{S},<) \rightarrow C M_{-}^{*}(\mathscr{S},<)$ defined by $B^{ \pm}\left(\Delta_{*}^{ \pm}(P), \phi_{*}^{ \pm}(I)\right)=\left(h^{-1} \circ \operatorname{Hom}\left(\Delta_{*}^{ \pm}(P)\right) \circ h, h^{-1} \circ \operatorname{Hom}\left(\phi_{*}^{ \pm}(I)\right) \circ h\right)$, where $h$ is the isomorphism of the cohomology universal coefficient theorem. As in Theorem $3.4, B^{+}$and $B^{-}$carry connection matrices to connection matrices. Thus the four collections of connection matrices are all naturally identified with each other, and the four homology index functors provide equivalent information about Morse decompositions.

In particular, they all generate equivalent Morse inequalities:

Corollary 3.5. The Morse inequalities generated by $\mathrm{CH}_{*}^{+}$and $\mathrm{CH}_{+}^{*}$ are equal; the Morse inequalities generated by $\mathrm{CH}_{*}^{-}$and $\mathrm{CH}_{-}^{*}$ are equal; the Morse inequalities generated by $\mathrm{CH}_{*}^{+}$and $\mathrm{CH}_{*}^{-}$are related by exchanging $t^{k}$ and $t^{n-k}$ in the Poincaré polynomials.

While the duality isomorphism exists for a large class of flows, and commutes with most of the basic structures of the Conley index theory, it does not hold in complete generality, nor preserve all of the index structures. Some examples illustrate these limitations.

In Example 1.2, the manifold $M$ is nonorientable, and the invariant set $S$ has integer (co)homology indices

$$
\begin{array}{cl}
C H_{*}^{+}(M ; S) \cong\left(0, \mathbf{Z}_{2}, \mathbf{Z}_{2}, 0, \ldots\right), & C H_{+}^{*}(M ; S) \cong\left(0, \mathbf{Z}_{2}, \mathbf{Z}_{2}, 0, \ldots\right), \\
C H_{*}^{-}(M ; S) \cong(\mathbf{Z}, \mathbf{Z} \oplus \mathbf{Z}, \mathbf{Z}, 0, \ldots), & C H_{-}^{*}(M ; S) \cong(\mathbf{Z}, \mathbf{Z} \oplus \mathbf{Z}, \mathbf{Z}, 0, \ldots),
\end{array}
$$

while the indices with $\mathbf{Z}_{2}$ coefficients are

$$
\begin{gathered}
C H_{*}^{+}(M ; S) \cong C H_{+}^{*}(M ; S) \cong\left(0, \mathbf{Z}_{2}, \mathbf{Z}_{2} \oplus \mathbf{Z}_{2}, \mathbf{Z}_{2}, 0, \ldots\right), \\
C H_{*}^{-}(M ; S) \cong C H_{-}^{*}(M ; S) \cong\left(\mathbf{Z}_{2}, \mathbf{Z}_{2} \oplus \mathbf{Z}_{2}, \mathbf{Z}_{2}, 0, \ldots\right) .
\end{gathered}
$$

Moreover, the ring structures with $\mathbf{Z}_{2}$ coefficients are

$$
\begin{gathered}
C H^{+}(M ; S) \cong\left(0,\langle a\rangle,\left\langle a^{2}, b\right\rangle,\left\langle a^{2} b\right\rangle, 0, \ldots\right), \\
C H^{-}(M ; S) \cong(\langle 1\rangle,\langle\alpha, \beta\rangle,\langle\alpha \beta\rangle, 0, \ldots) .
\end{gathered}
$$


This example illustrates several distinctions between the indices. First, the groups above are dual when $\mathbf{Z}_{2}$ coefficients are used, but not when $\mathbf{Z}$ coefficients are used, as $M$ is orientable over $\mathbf{Z}_{2}$, but not over $\mathbf{Z}$.

Second, the indices are dual as groups, but not as rings. The cohomology groups have distinct nontrivial ring structures, while their dual homology groups have no natural ring structure. That is, as groups, all four indices are essentially the same. As rings, they are all distinct. In particular, the forward and reverse cohomology groups have different cuplengths, and so may give different information in Ljusternik-Schnirelmann arguments, such as that used in [3].

Third, the duality isomorphism does not conjugate the maps induced by semiconjugacies (cf. [10]). That is, if $f: M^{\prime} \rightarrow M$ is a flow map, $S \subseteq M$ an isolated invariant set, the diagram may not commute:

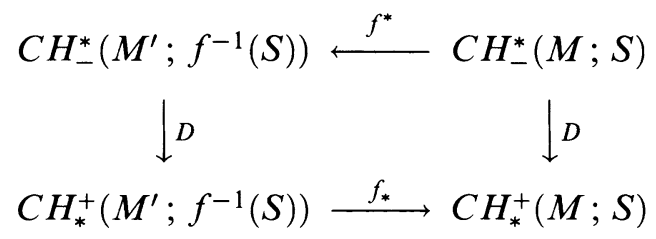

For example, in Example 1.2 take the inclusion $i: S \rightarrow M$. The homology Conley index of a set in itself is simply the homology of the set: $C H_{*}^{ \pm}(S ; S)=$ $H_{*}(S)$. In the reverse flow in Example 1.2, $S$ is an attractor, so its homology index is the Čech homology of the set: $C H_{*}^{-}(M ; S)=\check{H}_{*}(S)$. The inclusion is a proper semiconjugacy with $i_{*}: C H_{*}^{+}(S ; S) \rightarrow C H_{*}^{+}(M ; S)$ the zero map and $i^{*}: C H_{-}^{*}(S ; S) \rightarrow C H_{-}^{*}(M ; S)$ an isomorphism.

Finally, in the wedge of spheres $X=S^{1} \vee S^{2}$ in Example 1.3, the wedge point had homology indices $C H_{-}^{*}(X ; S)=(R, 0,0, \ldots)$ and $C H_{*}^{+}(X ; S)=$ $(0, R \oplus R, R, 0, \ldots)$. These are not dual, regardless of the ring $R$ chosen. That is, duality holds only for flows on manifolds.

\section{Applications}

Theorem 2.1 is essentially a closed result, establishing the hypotheses needed for the duality observed in Example 1.1 to hold for all isolated invariant sets. However, by adding another element to the Conley index theory which is both canonical and natural, the theorem raises the question of applications: does the duality isomorphism provide any dynamical information? Two modes of application are indicated in this section.

The first use of duality is that it can simplify computation of homology index groups. For example, if $S$ is isolated in $M$ and $C H_{*}^{ \pm}(\partial M ; S \cap \partial M)$ is nonzero, then $S \cap \partial M$ is nonempty, and remains so under continuation. Duality allows this to be observed without computing $C H_{*}^{+}(\partial M ; S \cap \partial M)$ directly.

Corollary 4.1. If $C H_{-}^{*}(M ; S)$ and $C H_{*}^{+}(M ; S)$ are not dual, then every isolated invariant set related by continuation to $S$ intersects $\partial M$, and has

$$
C H_{*}^{ \pm}(\partial M ; S \cap \partial M)
$$

nonzero. Further, if $\mathrm{CH}_{*}^{+}(M ; S)$ is nonzero, then $W^{u}(S)$ is not contained in $\partial \mathrm{M}$; if $\mathrm{CH}_{*}^{-}(M ; S)$ is nonzero, then $W^{S}(S)$ is not contained in $\partial M$.

In Examples 1.4, $\mathrm{CH}_{*}^{+}(M ; S)=0$, while $C H_{*}^{-}(M ; S) \cong(\mathrm{Z}, 0,0, \ldots)$. Thus $C H_{1}^{+}(\partial M ; S \cap \partial M) \cong C H_{2}^{+}(M, \partial M ; S) \cong \mathbf{Z}$, and $S \cap \partial M$ remains 
nonempty under continuation. Further, as $C H_{*}^{+}(M ; S)$ is nonzero, $W^{s}(S)$ is not contained in $\partial M$ and remains so under continuation. That is, $S$ and everything related to it by continuation must intersect the boundary and attract orbits from the interior.

A similar application of duality relates the stable and unstable sets of $S$. The stable and unstable sets of $S$ are $W^{s}(S)=\{x \in M: \omega(x) \subseteq S\}$ and $W^{u}(S)=\left\{x \in M: \omega^{*}(x) \subseteq S\right\}$ respectively. If $S$ is hyperbolic, these are immersed manifolds whose tangent bundles along $S$ are $T S \oplus E^{s}$ and $T S \oplus E^{u}$ respectively. If $\sigma^{s}$ and $\sigma^{u}$ are sections of $W^{s}(S) \backslash S$ and $W^{u}(S) \backslash S$ respectively, let $W_{\sigma}^{s}(S)=\left\{x \cdot \mathbf{R}^{-}: x \in \sigma^{s}\right\}, W_{\sigma}^{u}(S)=\left\{x \cdot \mathbf{R}^{+}: x \in \sigma^{u}\right\}$. For any two sections $\sigma^{s}, \tilde{\sigma}^{s}$, there exists a flow-defined homeomorphism between $\left(W_{\sigma}^{s}(S), \sigma^{s}\right)$ and $\left(W_{\tilde{\sigma}}^{s}(S), \tilde{\sigma}^{s}\right)$, so there are well-defined groups $H_{*}\left(W_{\sigma}^{s}(S), \sigma^{s}\right)$ and $H_{*}\left(W_{\sigma}^{u}(S), \sigma^{u}\right)$ independent of the section chosen. These groups find repeated use in relating the dynamics of the flow to the topology of the manifold. For instance, in Morse theory, the unstable sets form a CW decomposition of the manifold and these groups form the associated (co)chain complex.

If $\left(N, \varnothing, N_{1}, N_{2}\right)$ is an index quadruple, then $\sigma^{S}=N_{1} \cap W^{s}(S)$ and $\sigma^{u}=$ $N_{2} \cap W^{u}(S)$ are such sections. Further, sequences of quadruples $\left(N_{\alpha}, \varnothing, N_{1 \alpha}\right.$, $N_{2 \alpha}$ ) can be chosen such that $N_{1 \alpha} \cap W^{s}(S)=N_{1 \beta} \cap W^{s}(S)$ and $\cap_{\alpha} N_{\alpha}=W_{\sigma}^{s}(S)$. Thus $\check{H}_{*}\left(W_{\sigma}^{u}(S), \sigma^{u}\right) \cong C H_{*}^{+}(M ; S)$ and $\check{H}_{*}\left(W_{\sigma}^{s}(S), \sigma^{s}\right) \cong C H_{*}^{-}(M ; S)$, where $\check{H}$ denotes $\check{C}$ ech (co)homology. All of this is standard, and is used in the development of the Churchill sequence [1]. The new feature is that these groups are dual:

Corollary 4.2. There exists a duality isomorphism

$$
D: \check{H}^{k}\left(W_{\sigma}^{u}(S), \sigma^{u}\right) \rightarrow \check{H}_{n-k}\left(W_{\sigma}^{s}(S), \sigma^{s}\right)
$$

which is independent of the sections $\sigma^{s}$ and $\sigma^{u}$.

Note that this duality does not hold in general if singular homology is used. For example, there exists flow on $\mathbf{R}^{2}$ which have a Warsaw circle $S$ as an attractor. Then $\left(W_{\sigma}^{u}(S), \sigma^{u}\right)=(S, \varnothing)$, while $\left(W_{\sigma}^{s}(S), \sigma^{s}\right)=(A, \partial A)$, where $A$ is an annulus. Clearly, $H^{1}\left(W_{\sigma}^{u}(S), \sigma^{u}\right)$ and $H_{2-1}\left(W_{\sigma}^{s}(S), \sigma^{s}\right)$ are not dual.

These applications use only the fact that the duality map is an isomorphism, without using the precise form of the map. Another aspect of duality is to discover what information can be obtained by knowing which elements of $C H_{-}^{p}(M ; S)$ and $C H_{n-p}^{+}(M ; S)$ are identified by the map. Note that 2.1 and the naturality results help make the map computable: if it can be computed for one index quadruple, it is then computed for all others; if the set can be recognized as a sum or product, the map can be computed on the factors; if the map can be computed for one isolated invariant set, it is computed for all others related by continuation. However, the map is not "flow-defined." That is, the flow generates the maps $\phi_{+}, \phi^{-}, \theta$ used to show that $D$ is well defined, and the map $F_{\lambda, \mu}$ used to show that it is continuation-invariant, but the flow does not generate the map $D$ itself.

One way of using the map $D$ is to define from it a nonsingular pairing of $\mathrm{CH}_{*}^{-}(\mathrm{M} ; \mathrm{S})$ and $\mathrm{CH}_{*}^{+}(\mathrm{M} ; S)$, which can be thought of as an intersection pairing:

$$
C H_{p}^{-}(M ; S) \otimes C H_{n-p}^{+}(M ; S) \rightarrow \check{H}_{0}(S)
$$


Naturally related to it is an intersection pairing of $\check{H}_{*}\left(W_{\sigma}^{u}(S), \sigma^{u}\right)$ and $\check{H}_{*}\left(W_{\sigma}^{S}(S), \sigma^{S}\right)$. In [9], Kurland independently defines such intersection pairings and uses them to prove the existence of layers of solutions to certain boundary value problems.

Another intersection pairing related to duality involves attractor-repeller pairs. If $\left(A, A^{*}\right)$ is an attractor-repeller pair for $S$, then the set $C\left(A^{*}, A ; S\right)$ of connecting orbits from $A^{*}$ to $A$ is the intersection $W^{u}\left(A^{*}\right) \cap W^{s}(A) \cap S$. In [11], this intersection is measured by an intersection pairing $C H_{p}^{-}(M ; A) \otimes$ $C H_{q}^{+}\left(M ; A^{*}\right) \rightarrow \check{H}_{p+q-n-1}(C)$ where $C$ is a section of $C\left(A^{*}, A ; S\right)$. The duality isomorphism is used to show that this intersection pairing is related to the connection map $\partial: \mathrm{CH}_{q}^{+}\left(M ; A^{*}\right) \rightarrow \mathrm{CH}_{q-1}^{+}(M ; A)$. Namely, in the appropriate dimensions, an element $a \otimes b$ has nonzero intersection pairing if and only if $\partial b$ is a nonzero multiple of $D a$. Thus the connection map can be used to partially compute the topology of the connecting orbit set.

\section{REFERENCES}

1. C. Conley, Isolated invariant sets and the Morse index, CMBS Regional Conf. Ser. in Math., No. 38, Amer. Math. Soc., Providence, R. I., 1978.

2. C. Conley and J. Smoller, On the structure of magnetohydrodynamic shock waves, Comm. Pure Appl. Math. 27 (1974), 367-375.

3. C. Conley and E. Zehnder, On the Birkhoff-Lewis fixed point theorem and a conjecture of V. I. Arnold, Invent. Math. 73 (1983), 33-49.

4. $\ldots$, Morse-type index theory for flows and periodic solutions for Hamiltonian equations, Comm. Pure Appl. Math. 37 (1984), 207-253.

5. A. Dold, Lectures on algebraic topology, Springer-Verlag, Berlin, 1972.

6. R. Franzosa, Index filtrations and the homology index braid for partially ordered Morse decompositions, Trans. Amer. Math. Soc. 298 (1986), 193-213.

7. _ The connection matrix theory for Morse decompositions, Trans. Amer. Math. Soc. 311 (1989), 561-592.

8. H. Hattori and K. Mischaikow, On the existence of intermediate magnetohydrodynamic shock waves, preprint.

9. H. Kurland, Layers in singularly perturbed systems via homology continuation, preprint.

10. C. McCord, Mappings and homological properties in the Conley index theory, Ergodic Theory Dynamical Systems 8* (1988), 175-198.

11. _ Intersection pairings for attractor-repeller pairs, preprint.

12. J. Robbin and D. Salamon, Dynamical systems, shape theory and the Conley index, Ergodic Theory Dynamical Systems 8* (1988), 375-394.

13. D. Salamon, Connected simple systems and the Conley index of isolated invariant sets, Trans. Amer. Math. Soc. 291 (1985), 1-41.

14. E. Spanier, Algebraic topology, McGraw-Hill, New York, 1966.

Department of Mathematical Sciences, University of Cincinnati, Cincinnati, Ohio 